Network Working Group

Request for Comments: 2724

Category: Experimental
S. Handelman S. Stibler

IBM

N. Brownlee The University of Auckland

G. Ruth

GTE Internetworking

October 1999

\title{
RTFM: New Attributes for Traffic Flow Measurement
}

Status of this Memo

This memo defines an Experimental Protocol for the Internet community. It does not specify an Internet standard of any kind. Discussion and suggestions for improvement are requested. Distribution of this memo is unlimited.

Copyright Notice

Copyright (C) The Internet Society (1999). All Rights Reserved.

Abstract

The RTFM Traffic Measurement Architecture provides a general

framework for describing and measuring network traffic flows. Flows are defined in terms of their Address Attribute values and measured by a 'Traffic Meter'. This document discusses RTFM flows and the attributes which they can have, so as to provide a logical framework for extending the architecture by adding new attributes.

Extensions described include Address Attributes such as DscodePoint, SourceASN and DestASN, and Group Attributes such as short-term bit rates and turnaround times. Quality of Service parameters for Integrated Services are also discussed.

Table of Contents

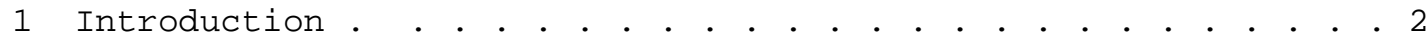

1.1 RTFM's Definition of Flows . . . . . . . . . . . . . . . . 3

1.2 RTFM's Current Definition of Flows and their Attributes . . 3 1.3 RTFM Flows, Integrated Services, IPPM and Research in Flows 4

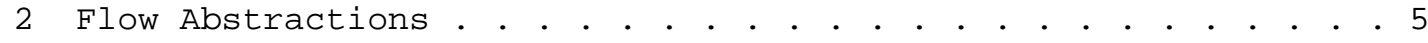

2.1 Meter Readers and Meters . . . . . . . . . . . . . 5

2.2 Attribute Types . . . . . . . . . . . . . . . . 6

2.3 Packet Traces . . . . . . . . . . . . . . . . . 7

2.4 Aggregate Attributes . . . . . . . . . . . . . . 8 
2.5 Group Attributes . . . . . . . . . . . . . . . 8

2.6 Actions on Exceptions . . . . . . . . . . . . . . . . . . . . . . . . .

3 Extensions to the 'Basic' RTFM Meter . . . . . . . . . . . . .10

3.1 Flow table extensions . . . . . . . . . . . . . . . . . .10

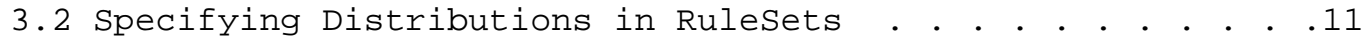

3.3 Reading Distributions . . . . . . . . . . . . . . . . . . . . . . . .

4 Extensions to the Rules Table, Attribute Numbers . • . . . .13

5 Security Considerations . . . . . . . . . . . . . . . . . . . 15

6 References . . . . . . . . . . . . . . . . . . . . . . . . . 16

7 Authors' Addresses . . . . . . . . . . . . . . . . . . . . . . . . . . .

8 Full Copyright Statement . . . . . . . . . . . . . . . . . . 18

1 Introduction

The Real-Time Flow Measurement (RTFM) Working Group (WG) has developed a system for measuring and reporting information about traffic flows in the Internet. This document explores the definition of extensions to the flow measurements as currently defined in [RTFM-ARC]. The new attributes described in this document will be useful for monitoring network performance and will expand the scope of RTFM beyond simple measurement of traffic volumes. A companion document to this memo will be written to define MIB structures for the new attributes.

This memo was started in 1996 to advance the work of the RTFM group. The goal of this work is to produce a simple set of abstractions, which can be easily implemented and at the same time enhance the value of RTFM Meters. This document also defines a method for organizing the flow abstractions to augment the existing RTFM flow table.

Implementations of the RTFM Meter have been done by Nevil Brownlee in the University of Auckland, NZ, and Stephen Stibler and Sig Handelman at IBM in Hawthorne, NY, USA. The RTFM WG has also defined the role of the Meter Reader whose role is to retrieve flow data from the Meter.

Note on flows and positioning of meters:

A flow as it traverses the Internet may have some of its characteristics altered as it travels through Routers, Switches, and other network units. It is important to note the spatial location of the Meter when referring to attributes of a flow. An example, a server may send a sequence of packets with a definite order, and inter packet timing with a leaky bucket algorithm. A meter reading downstream of the leaky bucket would record a set with minimal inter packet timing due to the leaky bucket. At the client's location, the packets may arrive out of sequence, with 
the timings altered. A meter at the client's location would record different attributes for the same flow.

\subsection{RTFM's Definition of Flows}

The RTFM Meter architecture views a flow as a set of packets between two endpoints (as defined by their source and destination attribute values and start and end times), and as BI-DIRECTIONAL (i.e. the meter effectively monitors two sub-flows, one in each direction).

Reasons why RTFM flows are bi-directional:

- The WG is interested in understanding the behavior of sessions between endpoints.

- The endpoint attribute values (the "Address" and "Type" ones) are the same for both directions; storing them in bidirectional flows reduces the meter's memory demands.

- 'One-way' (uni-directional) flows are a degenerate case. Existing RTFM meters can handle this by using one of the computed attributes (e.g. Flowkind) to indicate direction.

\subsection{RTFM's Current Definition of Flows and their Attributes}

Flows, as described in the "Architecture" document [RTFM-ARC] have the following properties:

a. They occur between two endpoints, specified as sets of attribute values in the meter's current rule set. A flow is completely identified by its set of endpoint attribute values.

b. Each flow may also have values for "computed" attributes (Class and Kind). These are directly derived from the endpoint attribute values.

c. A new flow is created when a packet is to be counted that does not match the attributes of an existing flow. The meter records the time when this new flow is created.

d. Attribute values in (a), (b) and (c) are set when the meter sees the first packet for the flow, and are never changed.

e. Each flow has a "LastTime" attribute, which indicates the time the meter last saw a packet for the flow. 
f. Each flow has two packet and two byte counters, one for each flow direction (Forward and Backward). These are updated as packets for the flow are observed by the meter.

g. ALL the attributes have (more or less) the same meaning for a variety of protocols; IPX, AppleTalk, DECnet and CLNS as well as $\mathrm{TCP} / \mathrm{IP}$.

Current flow attributes - as described above - fit very well into the SNMP data model. They are either static, or are continuously updated counters. They are NEVER reset. In this document they will be referred to as "old-style" attributes.

It is easy to add further "old-style" attributes, since they don't require any new features in the architecture. For example:

- Count of the number of "lost" packets (determined by watching sequence number fields for packets in each direction; only available for protocols which have such sequence numbers).

- In the future, RTFM could coordinate directly with the Flow Label from the IPv6 header.

\subsection{RTFM Flows, Integrated Services, IPPM and Research in Flows}

The concept of flows has been studied in various different contexts. For the purpose of extending RTFM, a starting point is the work of the Integrated Services WG. We will measure quantities that are often set by Integrated Services configuration programs. We will look at the work of the Benchmarking/IP Performance Metrics Working Group, and also look at the work of Claffy, Braun and Polyzos [C-B-P]. We will demonstrate how RTFM can compute throughput, packet loss, and delays from flows.

An example of the use of capacity and performance information is found in "The Use of RSVP with IETF Integrated Services" [IIS-RSVP]. RSVP's use of Integrated Services revolves around Token Bucket Rate, Token Bucket Size, Peak Data Rate, Minimum Policed Unit, Maximum Packet Size, and the Slack term. These are set by TSpec, ADspec and FLowspec (Integrated Services Keywords), and are used in configuration and operation of Integrated Services. RTFM could monitor explicitly Peak Data Rate, Minimum Policed Unit, Maximum Packet Size, and the Slack term. RTFM could infer details of the Token Bucket. The WG will develop measures to work with these service metrics. An initial implementation of IIS Monitoring has been developd at CEFRIEL in Italy [IIS-ACCT]. 
RTFM will work with several traffic measurements identified by IPPM [IPPM-FRM]. There are three broad areas in which RTFM is useful for IPPM.

- An RTFM Meter could act as a passive device, gathering traffic and performance statistics at appropriate places in networks (server or client locations).

- RTFM could give detailed analyses of IPPM test flows that pass through the Network segment that RTFM is monitoring.

- RTFM could be used to identify the most-used paths in a network mesh, so that detailed IPPM work could be applied to these most used paths.

2 Flow Abstractions Performance attributes include throughput, packet loss, delays,
jitter, and congestion measures. RTFM will calculate these
attributes in the form of extensions to the RTFM flow attributes
according to three general classes:

- 'Trace', attributes of individual packets in a flow or a segment of a flow (e.g. last packet size, last packet arrival time).

- 'Aggregate', attributes derived from the flow taken as a whole (e.g. mean rate, max packet size, packet size distribution).

- 'Group', attributes that depend on groups of packet values within the flow (e.g. inter-arrival times, short-term traffic rates).

Note that attributes within each of these classes may have various types of values - numbers, distributions, time series, and so on.

\subsection{Meter Readers and Meters}

A note on the relation between Meter Readers and Meters.

Several of the measurements enumerated below can be implemented by a Meter Reader that is tied to a meter with very short response time and very high bandwidth. If the Meter Reader and Meter can be arranged in such a way, RTFM could collect Packet Traces with time stamps and provide them directly to the Meter Reader for further processing. 
A more useful alternative is to have the Meter calculate some flow statistics locally. This allows a looser coupling between the Meter and Meter Reader. RTFM will monitor an 'extended attribute' depending upon settings in its Rule table. RTFM will not create any "extended attribute" data without explicit instructions in the Rule table.

\subsection{Attribute Types}

Section 2 described three different classes of attributes; this section considers the "data types" of these attributes.

Packet Traces (as described below) are a special case in that they are tables with each row containing a sequence of values, each of varying type. They are essentially 'compound objects' i.e. lists of attribute values for a string of packets.

Aggregate attributes are like the 'old-style' attributes. Their types are:

- Addresses, represented as byte strings (1 to 20 bytes long)

- Counters, represented as 64-bit unsigned integers

- Times, represented as 32-bit unsigned integers

Addresses are saved when the first packet of a flow is observed. They do not change with time, and they are used as a key to find the flow's entry in the meter's flow table.

Counters are incremented for each packet, and are never reset. An analysis application can compute differences between readings of the counters, so as to determine rates for these attributes. For example, if we read flow data at five-minute intervals, we can calculate five-minute packet and byte rates for the flow's two directions.

Times are derived from the Firstime for a flow, which is set when its first packet is observed. LastTime is updated as each packet in the flow is observed.

All the above types have the common feature that they are expressed as single values. At least some of the new attributes will require multiple values. If, for example, we are interested in inter-packet time intervals, we can compute an interval for every packet after the first. If we are interested in packet sizes, a new value is obtained as each packet arrives. When it comes to storing this data we have two options: 
- As a distribution, i.e. in an array of 'buckets'. This method is a compact representation of the data, with the values being stored as counters between a minimum and maximum, with defined steps in each bucket. This fits the RTFM goal of compact data storage.

- As a sequence of single values. This saves all the information, but does not fit well with the RTFM goal of doing as much data reduction as possible within the meter.

Studies which would be limited by the use of distributions might well use packet traces instead.

A method for specifying the distribution parameters, and for encoding the distribution so that it can be easily read, is described in section 3.2 .

\subsection{Packet Traces}

The simplest way of collecting a trace in the meter would be to have a new attribute called, say, "PacketTrace". This could be a table, with a column for each property of interest. For example, one could trace:

- Packet Arrival time (TimeTicks from sysUpTime, or microseconds from Firstime for the flow).

- Packet Direction (Forward or Backward)

- Packet Sequence number (for protocols with sequence numbers)

- Packet Flags (for TCP at least)

Note: The following implementation proposal is for the user who is familiar with the writing of rule sets for the RTFM Meter.

To add a row to the table, we only need a rule which pushpkts the PacketTrace attribute. To use this, one would write a rule set which selected out a small number of flows of interest, with a 'PushPkt PacketTrace' rule for each of them. A MaxTraceRows default value of 2000 would be enough to allow a Meter Reader to read one-second ping traces every 10 minutes or so. More realistically, a MaxTraceRows of 500 would be enough for oneminute pings, read once each hour.

Packet traces are already implemented by the RMON MIB [RMON-MIB, RMON2-MIB], in the Packet Capture Group. They are therefore a low priority for RTFM. 


\subsection{Aggregate Attributes}

RTFM's "old-style" flow attributes count the bytes and packets for packets which match the rule set for an individual flow. In addition to these totals, for example, RTFM could calculate Packet size statistics. This data can be stored as distributions, though it may sometimes be sufficient to simply keep a maximum value.

As an example, consider Packet Size. RTFM's packet flows can be examined to determine the maximum packet size found in a flow. This will give the Network Operator an indication of the MTU being used in a flow. It will also give an indication of the sensitivity to loss of a flow, for losing large packets causes more data to be retransmitted.

Note that aggregate attributes are a simple extension of the 'oldstyle' attributes; their values are never reset. For example, an array of counters could hold a 'packet size' distribution. The counters continue to increase, a meter reader will collect their values at regular intervals, and an analysis application will compute and display distributions of the packet size for each collection interval.

\subsection{Group Attributes}

The notion of group attributes is to keep simple statistics for measures that involve more than one packet. This section describes some group attributes which it is feasible to implement in a traffic meter, and which seem interesting and useful.

Short-term bit rate - The data could also be recorded as the maximum and minimum data rate of the flow, found over specific time periods during the lifetime of a flow; this is a special kind of 'distribution'. Bit rate could be used to define the throughput of a flow, and if the RTFM flow is defined to be the sum of all traffic in a network, one can find the throughput of the network.

If we are interested in '10-second' forward data rates, the meter might compute this for each flow of interest as follows:

- maintain an array of counters to hold the flow's 10-second data rate distribution.

- every 10 seconds, compute and save 10-second octet count, and save a copy of the flow's forward octet counter. 
To achieve this, the meter will have to keep a list of aggregate flows and the intervals at which they require processing. Careful programming is needed to achieve this, but provided the meter is not asked to do it for very large numbers of flows, it has been successfully implemented.

Inter-arrival times. The Meter knows the time that it encounters each individual packet. Statistics can be kept to record the interarrival times of the packets, which would give an indication of the jitter found in the Flow.

Turn-around statistics. Sine the Meter knows the time that it encounters each individual packet, it can produce statistics of the time intervals between packets in opposite directions are observed on the network. For protocols such as SNMP (where every packet elicits an answering packet) this gives a good indication of turn-around times.

Subflow analysis. Since the choice of flow endpoints is controlled by the meter's rule set, it is easy to define an aggregate flow, e.g. "all the TCP streams between hosts A and B." Preliminary implementation work suggests that - at least for this case - it should be possible for the meter to maintain a table of information about all the active streams. This could be used to produce at least the following attributes:

- Number of streams, e.g. streams active for n-second intervals. Determined for TCP and UDP using source-dest port number pairs.

- Number of TCP bytes, determined by taking difference of TCP sequence numbers for each direction of the aggreagate flow.

IIS attributes. Work at CEFRIEL [IIS-ACCT] has produced a traffic meter with a rule set modified 'on the fly' so as to maintain a list of RSVP-reserved flows. For such flows the following attributes have been implemented (these quantities are defined in [GUAR-QOS]): 
- QoSService:

- QoSstyle:

- QoSRate:

- QoSSlackTerm:

- QoSTokenBucketRate:

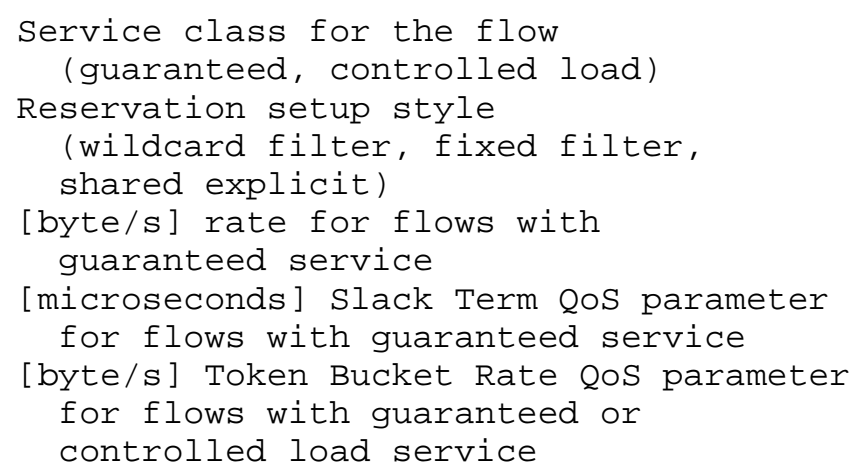

The following are also being considered:

- QoSTokenBucketSize: [byte] Size of Token Bucket

- QoSPeakDataRate: [byte/s] Maximum rate for incoming data

- QoSMinPolicedUnit: [byte] IP datagrams less than this are counted as being this size

- QoSMaxDatagramSize: [byte] Size of biggest datagram which conforms to the traffic specification

\subsection{Actions on Exceptions}

Some users of RTFM have requested the ability to mark flows as having High Watermarks. The existence of abnormal service conditions, such as non-ending flow, a flow that exceeds a given limit in traffic (e.g. a flow that is exhausting the capacity of the line that carries it) would cause an ALERT to be sent to the Meter Reader for forwarding to the Manager. Operations Support could define service situations in many different environments. This is an area for further discussion on Alert and Trap handling.

3 Extensions to the 'Basic' RTFM Meter

The Working Group has agreed that the basic RTFM Meter will not be altered by the addition of the new attributes of this document. This section describes the extensions needed to implement the new attributes.

\subsection{Flow table extensions}

The architecture of RTFM has defined the structure of flows, and this memo does not change that structure. The flow table could have ancillary tables called "Distribution Tables" and "Trace Tables," 
these would contain rows of values and or actions as defined above. Each entry in these tables would be marked with the number of its corresponding flow in the RTFM flow table.

Note: The following section is for the user who is familiar with the writing of rule sets for the RTFM Meter.

In order to identify the data in a Packet Flow Table, the attribute name could be pushed into a string at the head of each row. For example, if a table entry has "To Bit Rate" for a particular flow, the "ToBitRate" string would be found at the head of the row. (An alternative method would be to code an identification value for each extended attribute and push that value into the head of the row.) See section 4. for an inital set of ten extended flow attributes.

\subsection{Specifying Distributions in Rulesets}

At first sight it would seem neccessary to add extra features to the RTFM Meter architecture to support distributions. This, however, is not neccessarily the case.

What is actually needed is a way to specify, in a ruleset, the distribution parameters. These include the number of counters, the lower and upper bounds of the distribution, whether it is linear or logarithmic, and any other details (e.g. the time interval for short-term rate attributes).

Any attribute which is distribution-valued needs to be allocated a RuleAttributeNumber value. These will be chosen so as to extend the list already in the RTFM Meter MIB document [RTFM-MIB].

Since distribution attributes are multi-valued it does not make sense to test them. This means that a PushPkt (or PushPkttoAct) action must be executed to add a new value to the distribution. The oldstyle attributes use the 'mask' field to specify which bits of the value are required, but again, this is not the case for distributions. Lastly, the MatchedValue ('value') field of a PushPkt rule is never used. Overall, therefore, the 'mask' and 'value' fields in the Pushpkt rule are available to specify distribution parameters.

Both these fields are at least six bytes long, the size of a MAC address. All we have to do is specify how these bytes should be used! As a starting point, the following is proposed (bytes are numbered left-to-right. 


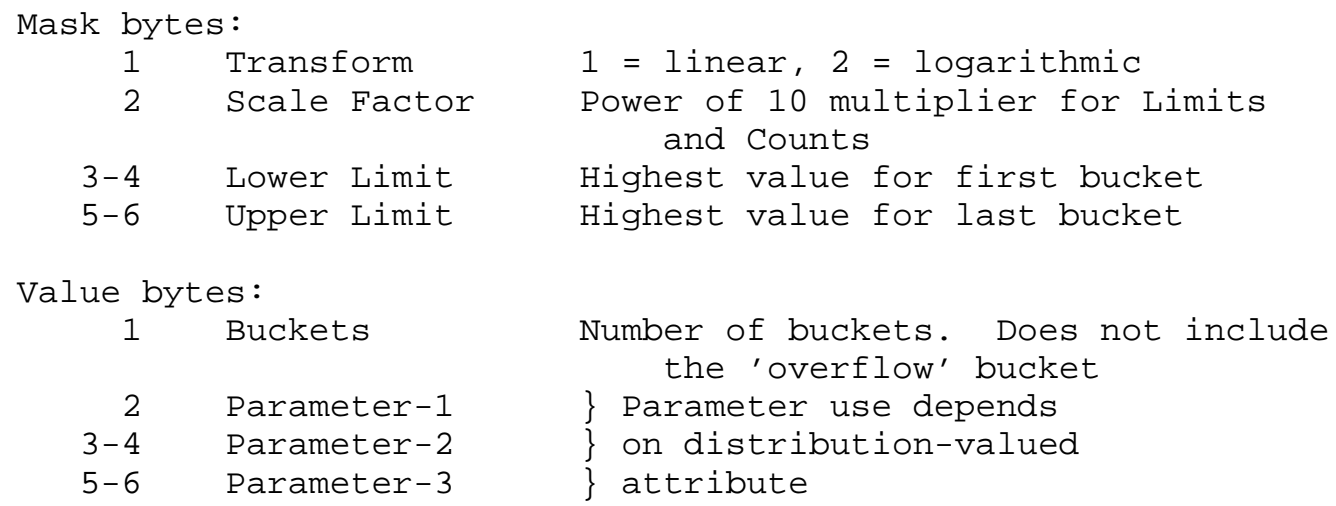

For example, experiments with NeTraMet have used the following rules:

$$
\begin{array}{lll}
\text { FrompacketSize } & \& 1.0 .25 ! 1500=60.0 ! 0: \quad \text { PushPkttoAct, Next; } \\
\text { ToInterArrivalTime } & \& 2.3 .1 ! 1800=60.0 .0 ! 0: \text { PushPkttoAct, Next; } \\
\text { FromBitRate } & \& 2.3 .1 ! 10000=60.5 .0 ! 0: \text { PushPkttoAct, Next; }
\end{array}
$$

In these mask and value fields a dot indicates that the preceding number is a one-byte integer, the exclamation marks indicate that the preceding number is a two-byte integer, and the last number is two bytes wide since this was the width of the preceding field. (Note that this convention follows that for IP addresses - 130.216 means $130.216 .0 .0)$.

The first rule specifies that a distribution of packet sizes is to be built. It uses an array of 60 buckets, storing values from 1 to 1500 bytes (i.e. linear steps of 25 bytes each bucket). Any packets with size greater than 1500 will be counted in the 'overflow' bucket, hence there are 61 counters for the distribution.

The second rule specifies an interarrival-time distribution, using a logarithmic scale for an array of 60 counters (and an overflow bucket) for rates from $1 \mathrm{~ms}$ to $1.8 \mathrm{~s}$. Arrival times are measured in microseconds, hence the scale factor of 3 indicates that the limits are given in milliseconds.

The third rule specifies a bit-rate distribution, with the rate being calculated every 5 seconds (parameter 1). A logarithmic array of 60 counters (and an overflow bucket) are used for rates from 1 kbps to $10 \mathrm{Mbps}$. The scale factor of 3 indicates that the limits are given in thousands of bits per second (rates are measured in bps). 
These distribution parameters will need to be stored in the meter so that they are available for building the distribution. They will also need to be read from the meter and saved together with the other flow data.

\subsection{Reading Distributions}

Since RTFM flows are bi-directional, each distribution-valued quantity (e.g. packet size, bit rate, etc.) will actually need two sets of counters, one for packets travelling in each direction. It is tempting to regard these as components of a single 'distribution', but in many cases only one of the two directions will be of interest; it seems better to keep them in separate distributions. This is similar to the old-style counter-valued attributes such as tooctets and fromoctets.

A distribution should be read by a meter reader as a single, structured object. The components of a distribution object are:

- 'mask' and 'value' fields from the rule which created the distribution

- sequence of counters ('buckets' + overflow)

These can be easily collected into a BER-encoded octet string, and would be read and referred to as a 'distribution'.

4 Extensions to the Rules Table, Attribute Numbers

The Rules Table of "old-style" attributes will be extended for the new flow types. A list of actions, and keywords, such as "ToBitRate", "Topacketsize", etc. will be developed and used to inform an RTFM meter to collect a set of extended values for a particular flow (or set of flows).

Note: An implementation suggestion.

Value 65 is used for 'Distributions', which has one bit set for each distribution-valued attribute present for the flow, using bit 0 for attribute 66, bit 1 for attribute 67, etc.

Here are ten possible distribution-valued attributes numbered according to RTFM WG consensus at the 1997 meeting in Munich:

ToPacketSize (66)

FromPacketsize (67) size of PDUs in bytes (i.e. number of bytes actually transmitted) 


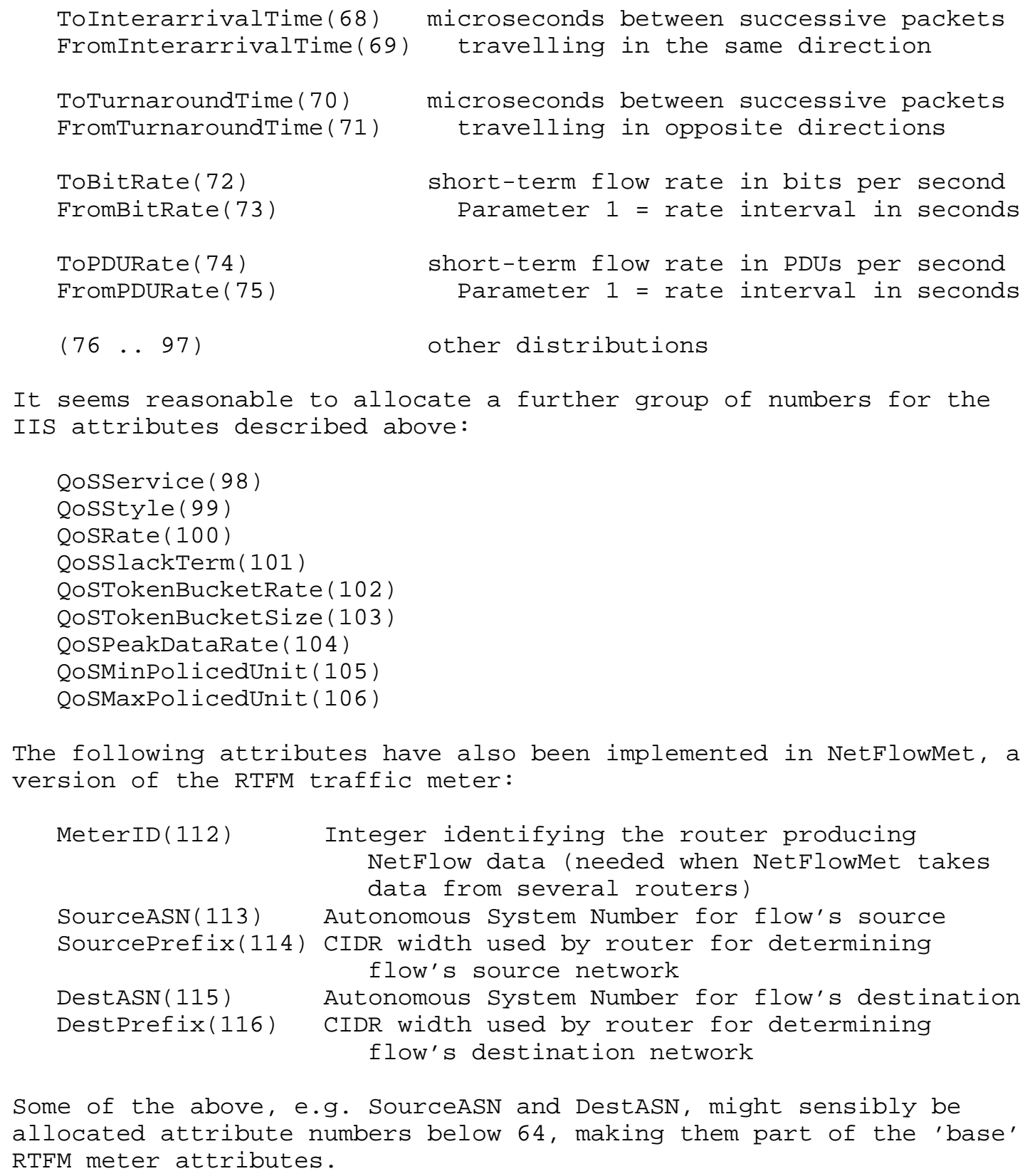

The following attributes have also been implemented in NetFlowMet, a version of the RTFM traffic meter:

MeterID(112) Integer identifying the router producing NetFlow data (needed when NetFlowMet takes data from several routers)

SourceAsN(113) Autonomous system Number for flow's source SourcePrefix(114) CIDR width used by router for determining flow's source network

DestASN (115) Autonomous System Number for flow's destination DestPrefix(116) CIDR width used by router for determining flow's destination network

Some of the above, e.g. SourceASN and DestASN, might sensibly be allocated attribute numbers below 64, making them part of the 'base' RTFM meter attributes. 
To support use of the RTFM meter as an 'Edge Device' for implementing Differentiated Services, and/or for metering traffic carried via such services, one more attribute will be useful:

DSCodepoint(118) DS Code Point ( 6 bits) for packets in this flow

Since the DS Code Point is a single field within a packet's IP header, it is not possible to have both Source- and Dest-CodePoint attributes. Possible uses of DSCodePoint include aggregating flows using the same Code Points, and separating flows having the same end-point addresses but using different Code Points.

5 Security Considerations

The attributes considered in this document represent properties of traffic flows; they do not present any security issues in themselves. The attributes may, however, be used in measuring the behaviour of traffic flows, and the collected traffic flow data could be of considerable value. Suitable precautions should be taken to keep such data safe. 
References

[C-B-P] Claffy, K., Braun, H-W, Polyzos, G., "A Parameterizable Methodology for Internet Traffic Flow Profiling," IEEE Journal on Selected Areas in Communications, Vol. 13, No. 8, October 1995 .

[GUAR-QOS] Shenker, S., Partridge, C. and R. Guerin, "Specification of Guaranteed Quality of Service", RFC 2212, September 1997 .

[IIS-ACCT] Maiocchi, S: "NeTraMet \& NeMaC for IIS Accounting: Users' Guide", CEFRIEL, Milan, 5 May 1998. (See also http: //www.cefriel.it/ntw)

[IIS-RSVP] Wroclawski, J., "The Use of RSVP with IETF Integrated Services", RFC 2210, September 1997.

[IPPM-FRM] Paxson, V., Almes, G., Mahdavi, J. and Mathis, M., "Framework for IP Performance Metrics", RFC 2330, May 1998 .

[RMON-MIB] Waldbusser, S., "Remote Network Monitoring Management Information Base", RFC 1757, February 1995.

[RMON2-MIB] Waldbusser, S., "Remote Network Monitoring Management Information Base Version 2 using SMIv2", RFC 2021, January 1997 .

[RTFM-ARC] Brownlee, N., Mills, C. and G. Ruth, "Traffic Flow Measurement: Architecture", RFC 2722, October 1999.

[RTFM-MIB] Brownlee, N., "Traffic Flow Measurement: Meter MIB", RFC 2720, October 1999. 
7 Authors' Addresses

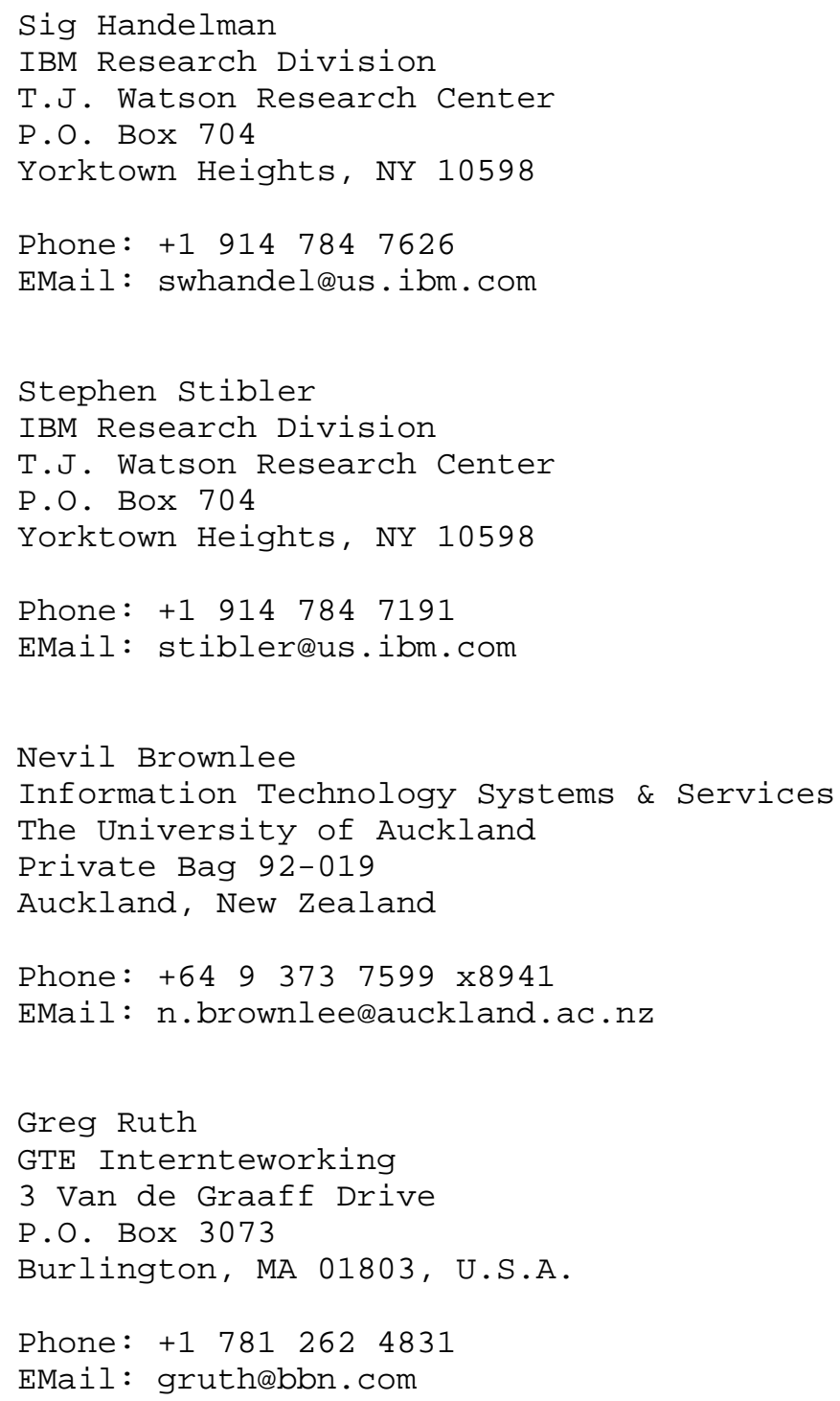


8. Full Copyright statement

Copyright (C) The Internet Society (1999). All Rights Reserved.

This document and translations of it may be copied and furnished to others, and derivative works that comment on or otherwise explain it or assist in its implementation may be prepared, copied, published and distributed, in whole or in part, without restriction of any kind, provided that the above copyright notice and this paragraph are included on all such copies and derivative works. However, this document itself may not be modified in any way, such as by removing the copyright notice or references to the Internet society or other Internet organizations, except as needed for the purpose of developing Internet standards in which case the procedures for copyrights defined in the Internet Standards process must be followed, or as required to translate it into languages other than English.

The limited permissions granted above are perpetual and will not be revoked by the Internet society or its successors or assigns.

This document and the information contained herein is provided on an "AS IS" basis and THE INTERNET SOCIETY AND THE INTERNET ENGINEERING TASK FORCE DISCLAIMS ALL WARRANTIES, EXPRESS OR IMPLIED, INCLUDING BUT NOT LIMITED TO ANY WARRANTY THAT THE USE OF THE INFORMATION HEREIN WILL NOT INFRINGE ANY RIGHTS OR ANY IMPLIED WARRANTIES OF MERCHANTABILITY OR FITNESS FOR A PARTICULAR PURPOSE.

Acknowledgement

Funding for the RFC Editor function is currently provided by the Internet society. 